\title{
A forme fruste of Shone's anomaly in a 65 year-old patient
}

\author{
Sherif E Moustafa*, Jacques Lesperance, Jean-Lucien Rouleau, Gilbert \\ Gosselin
}

\begin{abstract}
Shone's anomaly, a congenital cardiac malformation complex, consists of multiple levels of left heart obstruction. A rare case of an incomplete form of this anomaly discovered incidentally during cardiac catheterization for an unrelated event is described.
\end{abstract}

KEYWORDS: Congenital heart disease, Shone's anomaly

\section{INTRODUCTION}

In 1963, Shone et al. described a syndrome consisting of multilevel obstruction of all left heart structures. The original features of Shone's complex include mitral supra-annular ring, parachute mitral valve, subaortic stenosis, and coarctation of the aorta (CoA) (1).

In clinical practice, the definition of Shone's anomaly has been extended beyond the original Shone complex, to include patients with additional forms of left heart anomalies, such as mitral and aortic valvular lesions and supra-valvular aortic stenosis (SVAS). In the forme fruste, or incomplete form, some of the obstructions are present, whereas others are absent or only of minor relevance. We describe a 65-year-old male patient presenting with congestive heart failure (CHF). Cardiac catheterization revealed a variant of Shone's complex during routine coronary angiography to rule out atherosclerotic coronary artery disease (CAD).

\section{CASE PRESENTATION}

An active 65-year-old man was admitted to the hospital service with CHF. History was remarkable for CoA repair (at 11 years of age), sick sinus syndrome with permanent pacemaker implantation 8 years ago, diabetes mellitus and hypothyroidism. Twelve years ago, he presented with CHF. He underwent transthoracic echocardiogram (TTE) that revealed mild

*To whom correspondence should be addressed: Gilbert Gosselin

Montreal Heart Institute

5000 Belanger Street, Montreal, QC H1T 1C8, Canada

Email: gilbert.gosselin@icm-mhi.org apical and anterior wall segmental wall motion abnormalities (SWMA) and normal global ejection fraction (EF) of $50 \%$. Coronary arteries were free from significant atherosclerotic CAD at that time. He remained symptom free on antifailure medications until his recent hospitalization with severe CHF (III/IV NYHA classification). Cardiovascular examination revealed a systolic thrill over the base with a harsh 4/6 ejection systolic murmur best heard over the aortic areas, as well as a soft 3/6 apical holosystolic murmur. Jugular venous pressure was estimated at $18 \mathrm{mmHg}$. ECG showed atrial fibrillation, pacemaker spikes and left bundle branch block pattern. Laboratory data was notable for raised troponin $\mathrm{T} 1.6 \mathrm{ng} / \mathrm{mL}$ (normal $<0.03$ $\mathrm{ng} / \mathrm{mL}$ ) and creatine kinase-MB $51 \mathrm{ng} / \mathrm{mL}$ (normal < $6.2 \mathrm{ng} / \mathrm{mL}$ ).

Coronary angiography did not demonstrate significant atherosclerotic CAD. Selective left ventricle (LV) angiography and aortography revealed mildly dilated LV with severe systolic $(E F=15-20 \%)$ and diastolic (restrictive pattern) dysfunction, severe endomyocardial calcification (presumably secondary to fibroelastosis of both ventricles), multiple segmental wall motion abnormalities, severe subvalvular mitral calcification with mild valvular mitral stenosis (MS) (estimated mitral valve area $=2.0 \mathrm{~cm}^{2}$ ) and severe $(3 / 4)$ mitral regurgitation, bicuspid aortic valve (BAV) with moderately severe valvular aortic stenosis (AS) (estimated aortic valve area $=0.7 \mathrm{~cm}^{2}$ ), markedly dilated right ventricle with severe pulmonary hypertension $(75 / 40 / 55 \mathrm{mmHg}$ systolic/diastolic/mean pressure), mild SVAS without a gradient (Figure 1) and mild residual CoA without a gradient (Figure 2). TTE confirmed the aforementioned findings. In addition, it 


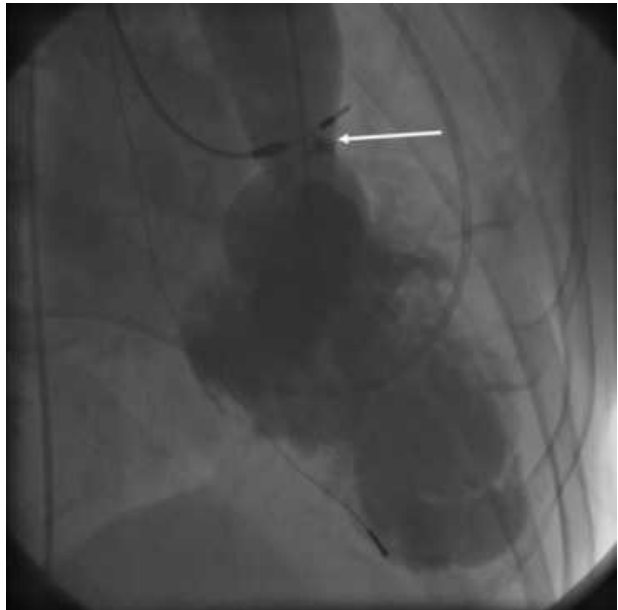

Figure 1: Left ventricular angiography in 1950 showing SVAS (arrow) and akinesia of the whole apex.

uncovered restricted calcified posterior papillary muscle. Dobutamine stress echocardiogram was negative for ischemia.

At that time, the suspected reason for CHF was myocarditis and the patient was followed up closely after hospital dismissal. Three months later, he returned for evaluation for surgical correction. Repeated cardiac catheterization revealed pulmonary vascular resistance of 11.4 which reduced to 4.4 wood's units (Normal range $=0.7-1.1$ wood's units) with decreased pulmonary pressure from $65 / 34 / 47$ to $29 / 13 / 20 \mathrm{mmHg}$ (systolic/diastolic/mean pressure) after Nitroprusside infusion. EF was $40-46 \%$ by TTE and Multiple Gated Acquisition Scan (MUGA). Decision was made for close follow up on antifailure measures including beta blocker (Bisoprolol), acetylsalicylic acid, angiotensin converting enzyme inhibitor (Perindopril) and simvastatin. Surgical correction was declined due to high risk and progressive improvement of EF. The patient's condition improved progressively with a stable EF of $40-45 \%$ during follow up in the heart function clinic. There were no major complications or hospitalizations during the follow up period.

\section{DISCUSSION}

In this report, we described a case that can be regarded as a forme fruste of Shone's anomaly including subvalvular mitral abnormalities, valvular mitral stenosis, bicuspid aortic valve, valvular aortic stenosis, supravalvular aortic stenosis and coarctation of the aorta with the absence of the supra-annular mitral ring. These anomalies tend to coexist, but the wide range of severity and predominance of each individual lesion may make optimal management troublesome. Even though the incomplete form of this anomaly is prone to be less symptomatic, it is nevertheless surprising that it

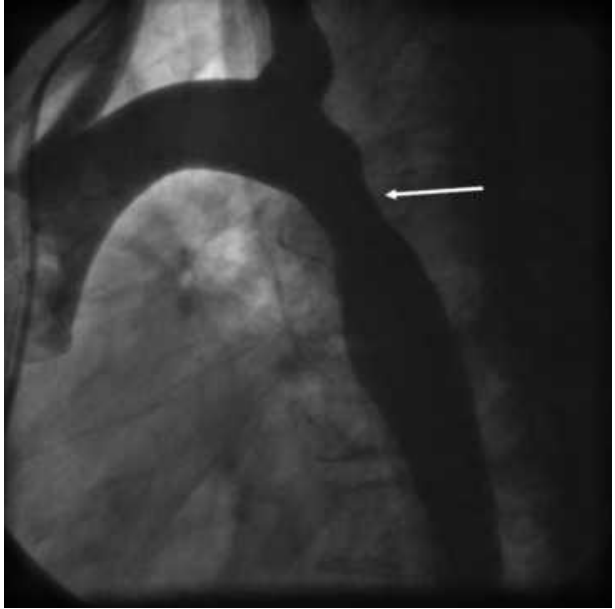

Figure 2: Aortogram in 2004 showing residual CoA repair site (arrow).

remained unnoticed in this patient until his mid-sixties. On their own, moderately severe AS and MS can be tolerated for a long time (2).

Our patient presented earlier in his life with $\mathrm{CoA}$ as the predominant outflow obstructive lesion. CoA may conceal the presence and potential hemodynamic severity of associated intracardiac lesions (3). Physicians were cautioned to be aware of other leftsided obstructive lesions that could be present in the symptomatic infant with CoA. The correlation of obstructive left-sided anomalies associated with CoA was confirmed by Becker et al. The authors believed that Shone's anomaly should be contemplated in those patients who have ongoing signs of CHF after operative repair of the CoA (4).

Shone et al. (1) noted that mitral valve obstruction appeared to be the most critical problem associated with the anomaly. Mitral commissurotomy alone was not adequate to relieve the obstruction. Bolling et al. confirmed the belief that severity of the mitral valve obstruction correlates inversely with long-term outcome and that operative mortality in patients with this anomaly is negatively influenced by the extent of mitral valve disease. The patients who demonstrated substantially elevated pulmonary artery pressures were those with the worst mitral obstruction and had, in general, the poorest outcome (5). In our case, MS was mild and this correlated well with the patient's longevity.

In conclusion, this rarely reported case of Shone's anomaly in an elderly patient draws attention to the importance of performing a meticulous search for other associated lesions in patients presenting early in life with CoA or any form of left ventricular outflow tract obstruction to prevent later presentation with end stage heart failure or myocardial disease. 


\section{REFERENCES}

1. Shone JD, Sellers RD, Anderson RC, Adams P Jr, Lillehei CW, Edwards JE. The developmental complex of "parachute mitral valve," supravalvular ring of left atrium, subaortic stenosis, and coarctation of aorta. Am J Cardiol. 1963;11:714-25.

2. Bonow RO, Braunwald E. Valvular Heart Disease. In Zipes, Libby, Bonow \& Braunwald, Braunwald's Heart Disease, 7th Edition: A Textbook of Cardiovascular Medicine. 2005 : 15531632.

3. Schwartz ML, Gauvreau K, Geva T. Predictors of outcome of biventricular repair in infants with multiple left heart obstructive lesions. Circulation. 2001;104: 682-7.

4. Becker AE, Becker MJ, Edwards JE. Anomalies associated with coarctation of aorta; particular reference to infancy. Circulation. 1970;41: 1067-75.

5. Bolling SF, Iannettoni MD, Dick M II, Rosenthal A, Bove EL. Shone's anomaly: operative results and late outcome. Ann Thorac Surg. 1990;49: 887-93.

Sherif E Moustafa, MD is an echocardiography fellow at the Sacre Coeur hospital in Montréal. He graduated from Cairo University in 1992 and received his Masters Degree (MSc) in Cardiology from the same university in 1997.

Jacques Lesperance, MD is a graduate of Laval University where he has obtained a Bachelor in Actuarial Science in 1974 and he is a Fellow of the Canadian Institute of Actuaries as well as the Society of Actuaries since 1979. He is currently a professor of Radiology at the Montreal Heart Institute.

Jean-Lucien Rouleau, MD is the Dean of the Faculty of Medicine, University of Montreal.

Gilbert Gosselin (M.D.C.M., C.S.P.Q., F.R.C.P.(C), FACC) is currently an assistant professor in interventional cardiology at the Montreal Heart Institute. He is also the head of the department of Cardiology and director of research at the Centre Hospitalier Pierre Le Gardeur in Montreal. 